\title{
Libertad de la voluntad y poderes causales
}

\author{
José TOMÁs Alvarado MARAmbio \\ Pontificia Universidad Católica de Valparaíso (Chile) \\ jose.alvarado.m@ucv.cl
}

\begin{abstract}
Resumen
Este trabajo discute una objeción bien conocida a la libertad de la voluntad libertaria en un mundo no determinista. En un mundo no determinista el estado de cosas total del mundo en un instante de tiempo $t$ es compatible con diferentes estados de cosas totales alternativos en el futuro de $t$. Se ha argumentado que, en cuanto son posibles diferentes alternativas a una decisión libre, es una cuestión de azar y suerte que tal decisión se ha tomado. Si una decisión libre es una cuestión de suerte, entonces el agente no puede ser considerado responsable por ella. Se argumenta que la dificultad aparece en una concepción anti-realista de la causalidad, donde los hechos causales son supervenientes a regularidades o dependencias contrafácticas. Una concepción realista de la causalidad puede, por ello, explicar cómo el agente está en control causal de la decisión libre tomada, cuando la decisión no cae bajo una regularidad o una dependencia contrafáctica. Una vez considerado cómo es que el agente está en control de la decisión, se argumenta que no se puede decir que la decisión libre es una cuestión de suerte para el agente.
\end{abstract}

Palabras claves: Libertad, determinismo, causalidad, dependencia contrafáctica, azar.

\section{Freedom of the will and causal powers}

\begin{abstract}
This paper discusses a well-known objection to libertarian free will in a non-deterministic world. In a nondeterministic world the complete state of affairs of the world in an instant of time $t$ is compatible with different alternative complete states of affairs in the future of $t$. It has been argued that, in so far as different alternatives are possible to a free decision, it is a matter of chance and luck that that decision is taken. If a free decision is a matter of luck, then the agent cannot be considered responsible for it. It is argued that the difficulty appears from an anti-realist conception of causality, where causal facts are supervenient on regularities or counterfactual dependences. A realist conception of causality can, then, explain how the agent is causally in control of the free decision taken when the decision does not fall under a regularity or a counterfactual dependence. Once considered how the agent is in control of the decision, it is argued that one cannot say that the free decision is a matter of luck for the agent.
\end{abstract}

Key words: Freedom, determinism, causality, counterfactual dependence, chance.

Profesor adjunto del Instituto de Filosofía de la Pontificia Universidad Católica de Valparaíso. Es autor de variados artículos en revistas especializadas sobre temas de metafísica modal y metafísica de propiedades como "Una teoría causal de la modalidad" (2009), "La función de los universales en metafísica modal" (2010) y "Clases de tropos como universales ersat?" (2011).

Este trabajo ha sido redactado en ejecución del proyecto de investigación 1090002 (CONICYT, Chile). Una versión preliminar fue presentada en el Congreso de Filosofía Cristiana, 20 al 22 de octubre de 2010, International Academy of Philosophy at Pontificia Universidad Católica de Chile. Agradezco los comentarios y sugerencias de los asistentes al Congreso. 


\section{Introducción}

Parte fundamental de nuestra comprensión de nosotros mismos como sujetos racionales, responsables moralmente por sus acciones y, por ello, responsables de la persona que somos, es que nuestra voluntad es libre. Esto es parte fundamental también de la forma en que se concibe la naturaleza humana en la doctrina cristiana ${ }^{1}$. Un debate tradicional es si esta libertad es compatible o no con el determinismo. No se pretende aquí discutir esta cuestión. Se asumirá la incompatibilidad de la libertad con el determinismo y, en particular, se asumirá que la libertad de la voluntad requiere la existencia de posibilidades abiertas para la acción. Esto es, sea $\mathrm{S}$ una variable que tiene como rango sujetos racionales y sea $\varphi$ una variable que tiene como rango acciones:

\section{(1) $\forall \mathrm{S} \forall \varphi[(\mathrm{S}$ hace libremente $\varphi) \rightarrow \diamond(\mathrm{S}$ no hace $\varphi)]$}

Hay múltiples refinamientos a esta tesis que no será necesario considerar aquí. La cuestión que interesa discutir en este trabajo, sin embargo, es la de la compatibilidad de la libertad de la voluntad con el indeterminismo. Si el mundo no es determinista, ¿en qué consiste la determinación que realiza la voluntad en el mundo al tomar, libremente, una decisión? Si un sujeto racional S decide libremente hacer $\varphi$, S debe en algún sentido que debe hacerse preciso- estar en «control» del llegar a realizarse $\varphi$. Si no está determinado que $S$ realizará $\varphi$, sin embargo, pareciera que el que llegue a realizarse $\varphi$ es una cuestión de azar, pues las cosas bien podrían haber sido diferentes. $\mathrm{Si} \varphi$ llega a realizarse por «azar», entonces no parece razonable decir que $S$ está en control del llegarse a realizar $\varphi$. Van Inwagen (2008: 461-462) resume la dificultad de este modo:

1 Dentro de los innumerables textos que podrían ser citados aquí, cfr. por ejemplo, el Concilio de Quiersy, año 853 (Denzinger, 1955: nn. 316-318); el III Concilio de Valence, año 855 (Denzinger, 1955: n. 322); la tesis 36 en la condenación de los errores de Martín Lutero de la Bula Exsurge domine, año 1520 (Denzinger, 1955: n. 776); el Decreto sobre la justificación del Concilio de Trento, año 1547, cap. 1 (Denzinger, 1955: n. 793); las tesis 27, 39, 65-67 en la condenación de los errores de Miguel du Bay de la Bula Ex omnibus afflictionibus, año 1567 (Denzinger, 1955: nn. 1955, 1027, 1039, 1065-1067, respectivamente). Por lo demás, en el Decreto de la Sagrada Congregación del Índice de 11 de junio de 1855 que condena el tradicionalismo se indica que la libertad del hombre se puede «probar con certeza» por el «razonamiento» (Denzinger, 1955: n. 1650). En el Concilio Vaticano II, la Constitución Pastoral Gaudium et Spes de 1965, confirmó esta doctrina tradicional en el n. 17. 
Si las leyes son indeterministas, entonces más de un futuro es, en efecto, consistente con aquellas leyes y el presente y pasado actuales. ¿Pero cómo puede nadie decidir cuál de esos futuros llega a ser actual? ¿No es acaso sólo una cuestión de azar (chance) cuál llega a ser actual? Si Dios 'retornase' un mundo indeterminista a su estado preciso en algún tiempo en el pasado y dejase que el mundo se desarrollase de nuevo, las cosas podrían acaecer de un modo diferente en la 'segunda' ocasión. Pero, entonces, si el mundo es indeterminista, ¿no es acaso simplemente una cuestión de azar cómo es que las cosas suceden de hecho en el único curso actual de eventos? Y si lo que hacemos es simplemente una cuestión de azar, bien, ¿quién llamaría a eso libertad?

En efecto, si el mundo es indeterminista, ¿cuál es la conexión entre el agente y la decisión libre? ¿Por qué el agente puede ser calificado como responsable de la acción? ¿Por qué puede ser el agente censurado moralmente o alabado moralmente por algo que depende de la «suerte»? La conexión entre la decisión libre y el agente de esa decisión ha sido comprendida de varias formas (cfr. Clarke, 2008): (i) se ha sostenido que la decisión libre no es causada, (ii) se ha sostenido también que la decisión libre es causada por un evento previo que involucra al agente; y (iii) se ha sostenido también que la decisión libre es causada directamente por el agente. En todos estos casos se ha replicado que no se explica cómo es que el agente está en control de la acción que se le imputa. Este trabajo pretende precisamente explorar esta cuestión y, en particular, considerar de qué modo una concepción causal y modal afincada en poderes causales podría servir para comprender mejor la naturaleza de la libertad de la voluntad en un mundo indeterminista.

Para esto, se considerará, en primer lugar, con más detenimiento la naturaleza del problema y cómo este depende - al menos en gran medida - de cómo sea comprendida la relación causal. En segundo lugar, se va a considerar la incidencia que pueden tener en la cuestión diferentes concepciones causales. En tercer lugar, se explorará cómo es que puede comprenderse la libertad de la voluntad en un mundo no determinista.

\section{1. ¿Incompatibilidad de la libertad con el indeterminismo?}

Sea como sea comprendido un mundo «determinista», el mundo es bien determinista o bien no determinista. Si la libertad de la voluntad es incompatible con el determinismo y, además, es incompatible con el indeterminismo, entonces, pareciera que se debiera concluir la 
incoherencia de la misma idea de libertad ${ }^{2}$. La incompatibilidad de la libertad de la voluntad con la indeterminación del mundo es fatal para el incompatibilista que también quiere defender la existencia de la libertad. Sería fatal también, por los mismos motivos, para la doctrina cristiana.

Determinismo es la tesis de que el mundo es determinista. Un mundo es determinista si es que, dado el estado total del mundo en un instante de tiempo $t_{1}$ (no necesariamente puntual) tal como se enuncia en una proposición $E_{1}$ y dadas todas las leyes naturales de ese mundo, tal como se enuncia en la proposición $\mathrm{N}$, se deduce todo estado de cosas total de ese mundo para todo instante de tiempo $t_{\mathrm{n}}\left(t_{\mathrm{n}}>t_{1}\right)$, esto es, todo instante de tiempo futuro respecto de $t_{1}$ (cfr. van Inwagen, 1983: 2-8; 1975: 47-49). Sea la proposición que enuncia el estado total del mundo en $t_{\mathrm{n}} \mathrm{E}_{\mathrm{n}}$. Entonces un mundo w es determinista si y sólo si:

(2) $E_{1}$ es verdadero en w; y

(3) $\mathrm{N}$ es verdadero en w; $\mathrm{y}$

(4) $\square \forall t_{\mathrm{n}}\left[\left(t_{\mathrm{n}}>t_{1}\right) \rightarrow\left(\left(\mathrm{E}_{1} \wedge \mathrm{N}\right) \rightarrow \mathrm{E}_{\mathrm{n}}\right)\right]$

El estado total del mundo w en $t_{1}$ junto con las leyes naturales de w «determinan» todo lo que acaece en el futuro respecto de $t_{1}$ en w. Aquí, el «estado de cosas total» de un mundo posible en un instante de tiempo $t$ es la suma mereológica de todos los estados de cosas efectivos en w en el instante $t$.

Una forma alternativa de concebir el carácter determinista de un mundo posible es haciendo apelación a estados de cosas duplicados (cfr. Lewis, 1983: 32-33). Un estado de cosas $s_{1}$ es un duplicado del estado de cosas $s_{2}$ si y sólo si todas y solo las propiedades intrínsecas ${ }^{3}$ instanciadas en $s_{1}$ se encuentran instanciadas en $s_{2}$. Dos estados de cosas duplicados entre sí son los que no difieren en propiedades intrínsecas. Un mundo posible $\mathrm{w}_{1}$ es determinista si es que todo otro mundo posible $\mathrm{w}_{2}$ que posea un segmento duplicado de $\mathrm{w}_{1}$ en el instante $t_{1}$ es duplicado de $\mathrm{w}_{1}$

2 En efecto, sea D la proposición de que el mundo es determinista, sea $\neg \mathrm{D}$ la proposición de que el mundo no es determinista y sea $\mathrm{L}$ la proposición de que la voluntad es libre. Se asume que $[\neg \diamond(\mathrm{D} \wedge \mathrm{L})]$, esto es $[\square(\mathrm{D} \rightarrow \neg \mathrm{L})]$. El problema que se considera es que $[\neg \vartheta(\neg \mathrm{D} \wedge \mathrm{L})]$, esto es $[\square(\neg \mathrm{D} \rightarrow \neg \mathrm{L})]$. Pero, por lógica modal, $[\square((\square(\mathrm{D} \rightarrow \neg \mathrm{L}) \wedge \square(\neg \mathrm{D} \rightarrow \neg \mathrm{L})) \rightarrow \neg \mathrm{L})]$.

3 Una propiedad $\mathrm{P}$ es intrínseca si y sólo si la posesión de $\mathrm{P}$ por un objeto a es indiferente a encontrarse el objeto $a$ solo o acompañado. Un objeto $x$ está solo en el mundo posible w si y sólo si no existe ningún otro objeto diferente de $x$ en w. Un objeto está acompañado si y sólo si no está solo (cfr. Lewis y Langton, 1998). 
para todo otro instante de tiempo $t_{\mathrm{n}}\left(t_{\mathrm{n}}>t_{1}\right)$. Los mundos posibles $\mathrm{w}_{1} \mathrm{y}$ $\mathrm{w}_{2}$ son duplicados en el segmento $t_{\mathrm{i}}$, por supuesto, si y sólo si, el estado de cosas total en $\mathrm{w}_{1}$ en $t_{\mathrm{i}}$ es duplicado del estado de cosas total en $\mathrm{w}_{2}$ en $t_{\mathrm{i}}$.

Un mundo no determinista debe tomarse, por lo tanto, como un mundo en el que el estado de cosas total del mundo en un instante de tiempo $t_{1}$ y las leyes naturales de ese mundo son compatibles con diferentes estados de cosas totales del mundo en los instantes de tiempo posteriores a $t_{1}$. Del mismo modo, un mundo w es no determinista si y sólo si hay otros mundos posibles que tienen segmentos iniciales con estados de cosas totales duplicados de w, pero en los que los estados de cosas totales posteriores al segmento inicial no son duplicados de w. Lo que acaece hasta un cierto instante de tiempo $t_{1}$ no determina lo que sucederá después.

Sea que el sujeto $S$ decide libremente realizar la acción $\varphi$ el instante $t_{1}$ en el mundo posible $\mathrm{w}_{1}$. El mundo posible $\mathrm{w}_{2}$, sin embargo, es un duplicado de $\mathrm{w}_{1}$ hasta el instante $t_{1}$, pero en $\mathrm{w}_{2} \mathrm{~S}$ no decide libremente realizar la acción $\varphi$. El que resulte la decisión de hacer $\varphi$ parece una cuestión de «suerte». Si uno está en una situación que es un duplicado del segmento de $\mathrm{w}_{1}$ hasta $t_{1}$, nada impide que pueda resultar que $\mathrm{S}$ decida hacer $\varphi$ o que $S$ decida no hacer $\varphi$. Si es una cuestión de azar o de «suerte» que $\mathrm{S}$ haya decidido hacer $\varphi$, ¿cómo es que $\mathrm{S}$ está en control de la decisión tomada? $\mathrm{Si}$ es por «suerte» que se ha tomado tal decisión, entonces no es una decisión de la que el agente pueda ser responsable. Señala Clarke (2008):

Si una decisión está causada de un modo no determinista, y si hay una posibilidad hasta antes de que ocurra de que el agente tome (en ese momento) una decisión diferente, entonces hay un mundo posible que es exactamente igual que el mundo actual hasta el momento de la decisión, pero en donde el agente toma la decisión alternativa. No hay nada, por lo tanto, en el agente con anterioridad a la decisión — de hecho, no hay nada en el mundo con anterioridad a ese momento- que explique la diferencia entre tomar una decisión y tomar la otra. Esta diferencia, entonces, es simplemente una cuestión de suerte. Y si la diferencia entre el hecho de que el agente tome una decisión y el hecho de que tome otra es simplemente una cuestión de suerte, el agente no puede ser responsable de la decisión que tome.

El problema consiste, entonces, en que parece haber una incompatibilidad entre estar un sujeto en control de una decisión y ser responsable por tal decisión, por una parte, y el ser esa decisión el 
resultado no determinado por lo que sucede con anterioridad al instante en que se toma la decisión, por otro lado. El desafío para quien quiera defender una teoría incompatibilista de la libertad de la voluntad (en donde la libertad sea coherente) es desarrollar una concepción que, (i) suponiendo el carácter no determinista del mundo, (ii) explique cómo es que el agente está en control de sus decisiones libres y es responsable por ellas. Sin reformas más profundas en nuestro modo de entender la naturaleza de la causalidad, sin embargo, no pareciera que puedan ser satisfechos estos requerimientos teóricos.

Supóngase que la causa de una decisión libre es un evento que involucra al agente de la decisión. Las condiciones de identidad de un evento se toman aquí como constituidas por un objeto, una propiedad y un instante de tiempo. Esto es, dos eventos $e_{1}$ y $e_{2}$ son el mismo evento si y sólo si constituyen la instanciación de la misma propiedad en el mismo objeto y en el mismo instante de tiempo (cfr. Kim, 1993: 33-52). Tradicionalmente se ha sostenido que los relata de la relación causal son eventos, por lo que esta concepción no es excesivamente revisionaria. La causa de que el agente $S$ decida hacer $\varphi$ en el instante $t_{2}$ será otro evento que consistirá - por lo menos- en la posesión por parte de $\mathrm{S}$ de alguna propiedad $\mathrm{P}$ en un instante de tiempo $t_{1}\left(t_{1} \leq t_{2}\right)$. En las ontologías de la causalidad tradicionales, al menos desde Hume, un evento $c$ causa el evento $e$ porque siempre que acaece un evento del tipo al que pertenece $c$ se sucede un evento del tipo al que pertenece $e$ o, al menos, la mayor parte de las veces en que sucede un evento del tipo al que pertenece $c$ le sigue la ocurrencia de un evento del tipo al que pertenece $e$. Es obvio, sin embargo, que nada de esto se podría aplicar aquí, si es que realmente $\mathrm{S}$ ha decidido libremente hacer $\varphi$ y este hecho implica que $S$ podría no haber decidido hacer $\varphi$. La conexión causal entre el evento de caer $S$ bajo $\mathrm{P}$ en $t_{1} \mathrm{y}$ el evento de decidir $\mathrm{S}$ hacer $\varphi$ no puede ser la conjunción constante entre el instanciar $\mathrm{P}$ y el decidir hacer $\varphi$. Tampoco puede ser que el caer bajo $\mathrm{P}$ hace más probable que se decida hacer $\varphi$ a que no se decida hacer $\varphi$. Si la decisión es realmente libre, aunque estadísticamente pueda asignarse una probabilidad condicional mayor a la decisión de no hacer $\varphi$ dada la instanciación de $\mathrm{P}$, el sujeto $\mathrm{S}$ podría decidir hacer $\varphi$ de todos modos. El evento causa no puede tomarse aquí como haciendo más probable la ocurrencia del efecto, por lo tanto.

La relación causal entre el instanciar $\mathrm{P}$ en $t_{1}$ y el decidir hacer $\varphi$ en $t_{2}$ tampoco se presta muy fácilmente para un análisis contrafáctico. En el análisis contrafáctico de la causalidad el evento $c$ causa el evento $e$ si y sólo si, si el evento $c$ no hubiese ocurrido, entonces el evento $e$ tampoco 
hubiese ocurrido (cfr. Lewis, 1973). El condicional contrafáctico en cuestión es verdadero en un mundo posible w si y sólo si en todos los mundos posibles más cercanos a w o bien $c$ ha ocurrido (el antecedente es falso) o bien $e$ no ha ocurrido (el consecuente es verdadero). El problema aquí es que, si la decisión de hacer $\varphi$ es libre, entonces no parece razonable sostener que si $\mathrm{S}$ no hubiese instanciado $\mathrm{P}$ en $t_{1}$ entonces $\mathrm{S}$ no hubiese decidido hacer $\varphi$ en $t_{2}$. Sea que la decisión libre se toma en $\mathrm{w}_{1}$. La forma en que se evalúa un condicional contrafáctico para analizar una relación causal en un caso como éste debería ser considerar lo que acaece en mundos posibles duplicados de $\mathrm{w}_{1}$ hasta el instante inmediatamente anterior a la ocurrencia de la causa, esto es, hasta antes de $t_{1}$. Se supone que no se instancia P en $t_{1}$ en esos mundos manteniendo todo lo demás idéntico. La relación causal debería consistir en que en ninguno de esos mundos se decide hacer $\varphi$. Esto es formalmente compatible con la existencia de alternativas abiertas en $t_{2}$ en que se decide no hacer $\varphi$ (habrá, pues, mundos posibles duplicados de $\mathrm{w}_{1}$ en el segmento temporal indicado que serán seguidos por la decisión de no hacer $\varphi$ ). Después de todo, lo que interesaría para el análisis contrafáctico no es que en todos los mundos (cercanos) en que se instancia $\mathrm{P}$ en $t_{1}$ se decida hacer $\varphi$ en $t_{2}$, -lo que chocaría frontalmente con el principio (1) de posibilidades abiertas indicado arriba- sino que basta con que en ninguno de los mundos (cercanos) donde no se instancia P en $t_{1}$ se decida hacer $\varphi$ en $t_{2}$. Si el evento de instanciar P en $t_{1}$ no determina la decisión de hacer $\varphi$ en $t_{1}$, sin embargo, no se ve cómo debería ser necesario para tal decisión.

Supóngase, en efecto, que la propiedad $\mathrm{P}$ en cuestión es una compleja propiedad estructural que especifica el estado mental del agente $\mathrm{S}$ en el instante $t_{1}$, esto es, especifica qué creencias y valoraciones posee el agente en ese instante. Es usualmente a esto a lo que se apela para explicar causalmente una acción libre. Se trataría de creencias como, por ejemplo, acerca de que $\varphi$ causaría $\psi$ y evaluaciones como, por ejemplo, que $\psi$ es bueno. Siendo el sujeto $S$ libre, sin embargo, puede suponerse un mundo posible en que existiendo estas mismas creencias y valoraciones se decide no hacer $\varphi$ y, en cambio, se decide hacer algo incompatible con $\varphi$, sea $\zeta$. La instanciación de la propiedad P que, en principio, podría tomarse como la base para explicar causalmente la decisión de hacer $\varphi$, parece resultar aquí la causa de otra decisión, la decisión de hacer $\zeta$. Puede suponerse que estadísticamente la probabilidad de que la instanciación de $\mathrm{P}$ sea seguida por la decisión de hacer $\varphi$ es alta y la probabilidad de sea seguida por la decisión de hacer $\zeta$ 
es baja. Esto no impide que se decida hacer $\zeta$, en efecto, porque el sujeto $\mathrm{S}$ es libre. Se puede suponer aquí que hay otra propiedad $\mathrm{Q}$ tal que estadísticamente la probabilidad de que su instanciación sea seguida por la decisión de hacer $\zeta$ sea alta y la probabilidad de que sea seguida por la decisión de hacer $\varphi$ sea baja. Tratándose de decisiones libres, nada parece impedir que el evento de instanciar Q en $t_{1}$ sea seguido por la decisión de hacer $\varphi$ en $t_{2}$ en vez de la decisión de hacer $\zeta$ en $t_{2}$, por el principio (1) de posibilidades abiertas. Si esto es así, entonces tampoco se puede suponer que la instanciación de $\mathrm{P}$ es necesaria para la decisión de hacer $\varphi$ y el análisis contrafáctico falla. Así como el principio de posibilidades abiertas implica que el mismo evento que causa la decisión de hacer $\varphi$, podría causar la decisión de hacer $\zeta$, también implica que otro evento cuya probabilidad de causar la decisión de hacer $\zeta$ sea alta, podría causar la decisión de hacer $\varphi$.

La cuestión aquí es que bajo cualquier teoría causal anti-realista, esto es, bajo cualquier teoría de la causalidad en donde ésta sea superveniente a otros hechos más básicos, tales como regularidades o dependencias contrafácticas, resulta muy difícil de explicar la conexión causal entre el agente (esto es, el evento que incluye al agente) y la decisión libre. Las cosas son incluso peores, sin embargo, si es que se proponen teorías más revisionarias para explicar la conexión entre el agente y la decisión libre. Una serie de propuestas han sostenido que la decisión libre es causada no por un evento que incluye al agente, sino por el agente. Es el agente y no un evento el relatum de la relación causal en que el efecto es la decisión libre (cfr., por ejemplo, Chisholm, 1964). Si uno pretende postular algún tipo de teoría anti-realista de la causalidad, como son las tradicionales teorías de la regularidad o las teorías contrafácticas, sin embargo, ¿en qué consiste la conexión causal entre el agente y la decisión libre? No tiene ningún sentido pensar que la existencia del agente $S$ sea seguida regularmente —o con una alta frecuencia- por la decisión de hacer $\varphi$. La regularidad, si es que existiese, no podría darse simplemente entre el agente y la decisión de hacer $\varphi$ en $t_{2}$, pues sería ridículo pensar que en cada instante de tiempo en que el agente existe ha de ser seguida su mera existencia por la decisión libre de hacer $\varphi$. La causa tendría que ser algo así como la existencia del agente $\mathrm{S}$ en el instante $t_{1}^{4}$. Es difícil pensar en

\footnotetext{
$4 \quad$ La existencia del agente $S$ en el instante $t_{1}$ se parece bastante a un evento y no a un mero objeto. Supongo que lo que se sostendría en un caso semejante es que la existencia no es una propiedad y que el trutbmaker de la proposición [S existe] no es la posesión por parte de $\mathrm{S}$ de la propiedad de la existencia, sino simplemente $\mathrm{S}$. No es necesario entrar en esta cuestión aquí, pero debe notarse que para quienes sostenemos
} 
alguna regularidad entre la mera existencia de $\mathrm{S}$ y la decisión libre de hacer $\varphi$. La mera existencia de un agente $S$ será indiferente a circunstancias en las que el agente crea que hacer $\varphi$ es malo y también circunstancias en las que crea que hacer $\varphi$ es bueno. Si alguna regularidad fuese a ser descrita aquí, debería - por lo menos- tomar en consideración factores como las valoraciones y las creencias de los agentes racionales, pero como lo único relevante aquí es la existencia del agente, nada de esto puede ser tomado en cuenta.

La dependencia contrafáctica entre la existencia del agente $\mathrm{S}$ en $t_{1} \mathrm{y}$ la decisión de hacer $\varphi$ en $t_{2}$ podría parecer aquí funcionar, pero es una dependencia contrafáctica «barata» que no parece fijar una dependencia ontológica muy fuerte. En efecto, si parte de las condiciones de identidad de la decisión libre de $S$ de hacer $\varphi$ es que se trata de una decisión de $S$, es trivial que en todos los mundos posibles en los que no exista $S$ no habrá decisión libre de $\mathrm{S}$ de hacer $\varphi$. Una dependencia contrafáctica más interesante, sin embargo, fácilmente falla. Lo que se debería pretender aquí es fijar la dependencia de la decisión de hacer $\varphi$ en ciertos estados mentales de creencia y valoración, o - si lo prefiere un fisicalista- en ciertos estados físicos del cerebro. Nada de esto puede aparecer, sin embargo, si es que aquello que se toma en consideración es la mera existencia del agente $\mathrm{S}$. Recuérdese que la relación causal aquí no se daría entre el estar el agente $S$ en tal o cual estado mental o en poseer tal o cual estado físico en su cerebro en un instante de tiempo (eventos, por supuesto), sino entre la mera existencia del agente $\mathrm{S}$ y su decisión libre.

Otros han postulado que no hay relación causal alguna entre el agente o entre un evento que incluye al agente y una decisión libre. Las decisiones libres son aquí sencillamente incausadas. Es ocioso todo comentario respecto a esta propuesta. No parece explicar de ningún modo cómo es que el agente está en control de la decisión tomada y ésta puede serle a él imputada como a quien es responsable por ella. Tampoco parecen ofrecer explicaciones satisfactorias las alternativas teóricas anteriores. El punto es que pareciera que, si se adopta de entrada una concepción anti-realista de la causalidad, no resulta explicable que el agente es responsable de sus decisiones libres, pues, si se trata de decisiones libres auténticas, los antecedentes causales que puedan ser aducidos para la decisión ni determinan, ni hacen más probable la decisión.

que hay una propiedad de existencia ontológicamente robusta que constituye a los objetos, no podemos sino ver aquí un evento. 


\section{Relaciones causales primitivas}

La conjetura fundamental de este trabajo es que los problemas explicativos no tienen que ver con cuál sea el relatum de la causalidad, sino con la concepción general que se tenga sobre la naturaleza de la relación causal. Para que pueda asignarse a un agente como responsable de una decisión libre y como en «control» de la decisión tomada, debe poder decirse con verdad que ese agente es causa de la decisión tomada o, por lo menos, que es integrante de la causa de la decisión tomada. Si se supone, sin embargo, que una decisión libre exige posibilidades alternativas de decisión, sin importar cuáles sean sus antecedentes, entonces no parece funcionar ninguno de los mecanismos usualmente aducidos para postular conexiones causales en las concepciones antirealistas de la causalidad, tal como se ha explicado. ¿Qué concluir de esta situación? Por supuesto, si uno considera absolutamente irrenunciable la postulación de alguna u otra teoría anti-realista de la causalidad, en la que las relaciones causales sean, por ejemplo, supervenientes a regularidades o a dependencias contrafácticas, entonces los problemas indicados son una razón para decretar la incoherencia de la noción de libertad, o para decretar la incoherencia de una concepción «libertaria» de la libertad en la que ésta es incompatible con el determinismo, o, por lo menos, para abandonar la cuestión como un 'misterio' del que no se conoce solución satisfactoria. Tal como en muchos otros casos, sin embargo, la situación dialéctica puede inclinar a reformas más profundas. Tal vez las dificultades tengan que ver con la forma en que se concibe la relación causal.

Supóngase, en primer lugar, que la relación causal no es ontológicamente superveniente a otros hechos, sea hechos acerca de regularidades de eventos, sea a hechos acerca de dependencias contrafácticas. Una relación causal puede, entonces, ocurrir de acuerdo a cierta regularidad, pero también puede ocurrir sin que su ocurrencia esté ligada a regularidad alguna. Una conexión puede perfectamente ser un evento altamente improbable. De un modo semejante, no será necesario esperar que las conexiones causales estén siempre fundadas en dependencias contrafácticas. Una decisión libre puede ser adoptada dadas ciertas creencias y valoraciones que la harían altamente improbable.

Se podría objetar aquí que una concepción semejante de la causalidad requiere una justificación independiente. Pienso, sin embargo, que los requerimientos de la libertad «libertaria» son suficiente justificación para motivar esta comprensión no reductiva de la causalidad. Esto es, la intuición de que somos libres y de que nuestra 
libertad implica posibilidades alternativas de decisión (esto es, la intuición de que parte de aquello en que consiste nuestra libertad es la existencia de posibilidades abiertas) es una razón para modificar nuestra comprensión de la relación causal, si es que las teorías tradicionales reductivas no concuerdan bien con ella. Afortunadamente existen motivaciones independientes que podrían ser aquí aducidas. No se pretende desarrollar todas las consideraciones que podrían apoyar la concepción de la causalidad como una relación de dependencia ontológica primitiva, pero sí mostrar una batería de argumentos que muestran que no se trata de una concepción extravagante.

(a) Supóngase que es una regularidad que toda instancia de un $F_{1}$ es seguida de una instancia de un $\mathrm{F}_{3}$ o bien seguida de una instancia de un $\mathrm{F}_{4}$, pero no ambos (cf. Tooley, 1987: 199-202). Supóngase que también es una regularidad que toda instancia de un $\mathrm{F}_{2}$ es seguida de una instancia de un $\mathrm{F}_{3}$ o bien seguida de una instancia de un $\mathrm{F}_{4}$, pero no ambos. Supóngase que, de acuerdo a los estándares del anti-realista, la secuencia de un $\mathrm{F}_{1}$ seguida por un $\mathrm{F}_{3}$ o seguida por un $\mathrm{F}_{4}$, y que la secuencia de un $\mathrm{F}_{2}$ seguida por un $\mathrm{F}_{3} \mathrm{O}$ seguida por un $\mathrm{F}_{4}$ constituyen relaciones causales. Supóngase que las intuiciones ordinarias acerca de tales secuencias - esto es, no «contaminadas» por argumentación filosófica- es que hay ahí auténticas conexiones causales. Pues bien, sea que el objeto $a$ instancie en $t_{1}$ las propiedades $F_{1}$ y $F_{2}$ y que este evento sea seguido por la instanciación en $t_{2}$ de las propiedades $\mathrm{F}_{3} \mathrm{y} \mathrm{F}_{4}$ por el mismo objeto $a$. $\mathrm{Si}$ fuese correcta alguna teoría anti-realista regularista o contrafáctica, entonces no estaría objetivamente determinado si el evento de instanciarse $\mathrm{F}_{1}$ en $a$ en $t_{1}$ ha causado el instanciarse de $\mathrm{F}_{3}$ en $a$ en $t_{2} \mathrm{o}$ ha causado el instanciarse de $\mathrm{F}_{4}$ en $a$ en $t_{2}$. No puede causar ambos eventos. Lo mismo sucede con el evento de instanciarse $\mathrm{F}_{2}$ en $a$ en $t_{1}$. Nuestra intuición, sin embargo, es que - si se trata de relaciones causales realesdebe haber un hecho determinado aquí acerca de qué causa qué. Si ni las regularidades ni las dependencias contrafácticas pueden hacer aquí tal discriminación, entonces pareciera que los hechos causales no son hechos acerca de regularidades o dependencias contrafácticas.

(b) Sea un mundo posible $\mathrm{w}$ en donde hay dos regiones indiscernibles cualitativamente entre sí, sean A y B, en cada una de las cuales se da una secuencia de eventos en el tiempo (cf. Tooley, 1987: 194-197). Cada objeto y cada evento en una de las regiones tendrá su duplicado en la otra región. Supóngase que en la región A hay un evento $e_{1}$ que causa otro evento $e_{2}$ de acuerdo a los estándares del anti-realista (sea por la existencia de una regularidad, sea por la existencia de una 
dependencia contrafáctica). Dada la configuración del mundo posible w existirán también en la región $\mathrm{B}$ dos eventos $e_{1}{ }^{*}$ y $e_{2}{ }^{*}$, duplicados respectivamente de $e_{1}$ y $e_{2}$ en la región A. Como todo lo relevante para que exista causalidad en una concepción anti-realista es qué distribución de propiedades intrínsecas y relaciones externas sean efectivas, y en la región $\mathrm{B}$ se da exactamente la misma distribución de propiedades intrínsecas y relaciones externas que en la región $\mathrm{A}$, deberá suponerse que hay una relación causal entre $e_{1}^{*} \mathrm{y} e_{2}^{*}$. Nuestra intuición aquí es que, obviamente, los eventos que están contiguos entre sí son aquellos que pueden estar conectados causalmente. En las concepciones anti-realistas, la causalidad entre dos eventos es un hecho que superviene a la distribución de propiedades que configuren una regularidad o una dependencia contrafáctica. ¿Por qué debe suponerse, entonces, que el evento $e_{1}$ causa el evento $e_{2}$ y no más bien el evento $e_{2}{ }^{*}$ ? Lo mismo podría sostenerse respecto del evento $e_{1}{ }^{*}$. ¿Por qué este evento debe verse como causando $e_{2}^{*}$ y no más bien $e_{2}$ ? No hay nada por lo que uno pueda excluir aquí relaciones causales trocadas entre $e_{1} \mathrm{y} e_{2}{ }^{*}$, como entre $e_{1}^{*}$ y $e_{2}$. Si las teorías anti-realistas de la causalidad tienen esta consecuencia abiertamente contra-intuitiva, entonces deben ser rechazadas.

(c) Sea un mundo posible $\mathrm{w}_{1}$ en el que se da una secuencia de eventos en el tiempo y en dónde, de acuerdo a los estándares del antirealista, quedan determinadas ciertas relaciones causales dadas las regularidades que son efectivas en $\mathrm{w}_{1} \mathrm{o}$ dadas las dependencias contrafácticas que puedan ser determinadas en $\mathrm{w}_{1}$, según qué otros mundos posibles resulten más cercanos a $\mathrm{w}_{1}$ y según lo que suceda en tales mundos. Resultará en $\mathrm{w}_{1}$, por ejemplo, que el evento $e_{1}$ será causa del evento $e_{2}$. Supóngase ahora un mundo posible $\mathrm{w}_{2}$ cualitativamente indiscernible respecto de $\mathrm{w}_{1}$ pero en el que el orden temporal de los eventos ha sido invertido (cfr. Tooley, 1987: 197-199). Esto es, el último evento de $\mathrm{w}_{1}$ es en $\mathrm{w}_{2}$ el primer evento, y el primer evento de $\mathrm{w}_{1}$ es en $\mathrm{w}_{2}$ el último evento. No hay nada más, por otro lado, por lo que los eventos de $\mathrm{w}_{1} \mathrm{y} \mathrm{w}_{2}$ puedan ser diferenciados entre sí. ¿Debe decirse también en $\mathrm{w}_{2}$ que $e_{1}$ causa $e_{2}$ ? Supóngase que los dos mundos posibles son perfectamente newtonianos, lo que acentúa la simetría entre las secuencias temporales. Si se sostuviera que la dirección del tiempo es superveniente a la dirección de la causalidad, no podría admitirse la existencia de un mundo posible en el que la secuencia de eventos esté invertida temporalmente, aunque se preserven las relaciones causales, pues si en $\mathrm{w}_{2}$ el evento $e_{1}$ causa el evento $e_{2}$, entonces $e_{1}$ debe ser temporalmente anterior a $e_{2}$. La dependencia de la dirección del tiempo en las relaciones causales es, sin embargo, una tesis controvertida. Si se 
rechaza esta dependencia, no habría ningún criterio para rechazar la coherencia del mundo posible $\mathrm{w}_{2}$ en donde todas las relaciones causales serán retrospectivas, si es que se asume alguna concepción anti-realista de la causalidad. Si las teorías anti-realistas tienen esta consecuencia fuertemente contra-intuitiva, entonces deben ser rechazadas.

(d) Un cuarto motivo de un carácter más general son las múltiples dificultades que enfrentan y han enfrentado las teorías anti-realistas de la causalidad. Pese a los esfuerzos reiterados de cohortes de filósofos, no se han logrado soluciones satisfactorias para explicar la causalidad por omisión, los fenómenos de prevención y de sobre-determinación causal (para una muestra de estas dificultades, cfr. Collins, Hall \& Paul, 2004). Puede ser, por supuesto, que un nuevo epiciclo explicativo consiga cuadrar todos los requerimientos sistemáticos, pero también es perfectamente posible que eso nunca suceda porque sencillamente la causalidad no sea un hecho reductible a otros hechos ontológicamente más básicos que ella. El fracaso reiterado de los intentos de solución es, por esto, un motivo para pensar en una concepción alternativa. Este motivo, por sí solo, podría verse no demasiado fuerte, pero si se considera en conjunto con los argumentos anteriores, parece razonable pensar en la causalidad como una relación primitiva de dependencia ontológica.

Siendo las cosas así, por lo tanto, no debe causar demasiada inquietud el que la causalidad que debería ser desplegada por un agente racional y libre al tomar una decisión no parezca poder ser capturada por las concepciones usuales anti-realistas de la causalidad. Si la causalidad no es superveniente ni a regularidades, ni a dependencias contrafácticas, entonces no habrá dificultades en ver al agente, o a un evento que integre como elemento a tal agente, causando sus decisiones libres y, por lo tanto, en «control» de la decisión tomada y responsable por ella.

\section{Suerte}

El desafío, sin embargo, no es simplemente explicar cómo es que el agente es causa de sus decisiones libres. Se ha sostenido que una decisión libre en un mundo indeterminista sería una cuestión de «suerte»y, por ello, no podría decirse que es el resultado de la decisión de un sujeto. Ésta, en realidad, pareciera una objeción contra cualquier relación causal en un mundo indeterminista. En efecto, la intuición sería que, si un evento $c$ causa un evento $e$, el evento $c$ debería «determinar» la ocurrencia 
de e. «Determinar» aquí tendría que tomarse como «hacer necesario que» o, por lo menos, «hacer más probable que», pero es obvio que si hay indeterminismo, no todos los procesos causales hacen necesaria o más probable la ocurrencia del efecto. Si el mismo evento $c$ causa finalmente la ocurrencia de un efecto improbable, entonces surgirá el mismo problema que ha sido explicado para hacer inteligible esta relación causal. Es irrelevante que aquí se trate o no de una decisión libre. Si éste es el problema, entonces la postulación de una concepción de la causalidad en la que ésta no haga necesaria ni más probable la ocurrencia del efecto, tal como se ha hecho arriba, es suficiente respuesta.

Será útil, de todos modos, considerar de manera específica esta cuestión. ¿Por qué la «suerte» parece excluir la libertad? Supóngase un agente $S$ que quiere conseguir la ocurrencia de $\varphi$. Para ello hace $\psi$. La probabilidad de que suceda $\varphi$ dado $\psi$ es extremadamente baja, 0,001, por ejemplo, y esta probabilidad condicional es conocida por S. Contra las probabilidades, sin embargo, ocurre $\varphi$. No parece que en este caso pueda decirse que $S$ ha hecho libremente $\varphi$, pues no era un resultado esperable razonablemente de la acción. En un caso semejante diríamos que el sujeto $\mathrm{S}$ obtuvo $\varphi$ por suerte. Si la probabilidad de que suceda $\varphi$ dado $\psi$ fuese de 0,6 ó 0,5, sin embargo, cambiaría este veredicto. También cambiaría si es que el sujeto $\mathrm{S}$, manteniendo la probabilidad condicional de $\varphi$ dado $\psi$ en 0,001 , hubiese hecho $\psi$ diez mil veces seguidas para obtener $\varphi$ como resultado. Hay aquí cierta vaguedad, naturalmente, pero esto no impide que puedan considerarse casos claros en los que la acción no puede ser atribuida a un sujeto porque la probabilidad de que acaezca es muy baja.

De todos modos, no puede sostenerse que la posibilidad de que no ocurra el resultado previsto impide que el agente esté en control de la acción, si es que la probabilidad de que no ocurra ese resultado es muy baja. Si la probabilidad de que no ocurra el resultado es alta, sin embargo, disminuye el control que tiene el agente sobre la acción y ya no puede imputársele el resultado. Así, si el resultado buscado es considerado algo bueno, el éxito del agente en conseguir ese resultado es objeto de alabanza si es que la probabilidad de conseguirlo es alta; si la probabilidad es baja, en cambio, no se alaba al agente, sino que se celebra su fortuna. Nadie es alabado por ganar una lotería, pero sí se congratula al ganador por su suerte. Del mismo modo, si el resultado es malo y la probabilidad de conseguir ese resultado es alta, el agente es objeto de reproche, pero si la probabilidad es muy baja, entonces el resultado se considera un infortunio. 
Se trataría aquí de aplicar este tipo de consideraciones pero a todas las decisiones libres en un mundo indeterminista. Tal como se ha visto, el control del agente no se ve mermada en los casos en los que, no estando determinado el resultado, es altamente probable. El problema seguiría en pie para el caso de decisiones libres que son altamente improbables dados sus antecedentes causales. Hay decisiones de este tipo que deben ser admitidas, si se acepta una concepción libertaria de la libertad. ¿En qué sentido las decisiones libres improbables escapan al «control»? Creo que escaparían al control de Dios o, en fin, de un demonio laplaciano que pretendiera disponer todo el curso de acontecimientos en un mundo que contiene agentes libres. En un mundo semejante, las decisiones libres improbables no podrían ser previstas y no podrían ser imputadas a quien haya pretendido disponer el curso de ese mundo. El que se produzcan es, para tal agente, simplemente buena o mala fortuna.

La cuestión ulterior aquí es si también puede decirse que para el agente de la decisión libre, esa decisión hubiese ocurrido por buena o mala fortuna. Creo que no. Supóngase que el sujeto $S$ decide libremente hacer $\varphi$ en $t_{2}$. En $t_{1}$ el sujeto $\mathrm{S}$ instancia la propiedad $\mathrm{P}$ (que consiste en el estado de mental de tener ciertas creencias y hacer ciertas valoraciones). La probabilidad de que se decida hacer $\varphi$ dada la instanciación de P es muy baja. Este caso sería análogo a los anteriores si es que el sujeto $\mathrm{S}$ hubiese pretendido provocar la decisión libre de hacer $\varphi$, haciendo que se instancie P. En este caso, no se podría decir que $\mathrm{S}$ está en control de la decisión tomada. No es esto lo que sucede, sin embargo. El sujeto $\mathrm{S}$ no ha decidido provocar la instanciación de $\mathrm{P}$ para luego provocar la decisión de hacer $\varphi$. S simplemente ha decidido hacer $\varphi$ y es libre al hacerlo porque podría no haberlo hecho. ¿Qué es lo que en esta situación hace menor el control que $\mathrm{S}$ tiene de la decisión tomada? ¿Importa que $S$ no pudo prever que tomaría tal decisión? ¿Importa que no pudo preverla un observador externo que conociese que $S$ se encontraba en el estado mental $\mathrm{P}$ en $t_{1}$ ? No veo que nada de esto sea relevante. Lo único relevante es que $\mathrm{S}$ es causa de la decisión libre tomada y nada impide esto. Si es causa de esa decisión, está en control de su ocurrencia y es responsable por ella.

\section{Conclusiones}

Se ha discutido aquí una dificultad dirigida contra las concepciones libertarias de la libertad, esto es, las concepciones en donde la libertad de 
la voluntad implica que toda decisión libre podría no haber sido tomada. $\mathrm{Si}$ un mundo posible no es determinista, entonces, dado el estado de cosas total de ese mundo en un instante de tiempo $t$, es posible que diferentes estados de cosas totales sean efectivos en ese mundo en los instantes futuros respecto de $t$. Dado el estado de cosas total del mundo en $t$, podría darse en el futuro que se decida libremente que $\varphi$ o que no se decida libremente que $\varphi$. El que se decida libremente que $\varphi$ parece, entonces, una cuestión de suerte. Si es por suerte que se ha decidido libremente hacer $\varphi$, entonces el sujeto $S$ no parece estar en control de la decisión y, por ello, no debería ser responsable por ella. Si el sujeto no está en control de la acción, entonces no parece que pueda decirse que se trata de una decisión libre. Pareciera, por lo tanto, que la libertad es incompatible con el indeterminismo. Si la libertad también es incompatible con el determinismo, entonces se mostraría aquí la incoherencia de la noción de libertad.

Se discute, en primer término, porqué el indeterminismo haría que el sujeto de una decisión libre no pueda decirse en «control» de la acción. Esta argumentación tiene mucho que ver con concepciones anti-realistas de la causalidad en donde ésta es superveniente a regularidades de eventos o a la dependencia contrafáctica de un evento respecto de otro. Desde la perspectiva de estas concepciones de la causalidad, es muy difícil explicar cómo es que el sujeto ha sido causa de la decisión tomada y, con ello, es muy difícil explicar cómo es que el sujeto está en control de la decisión tomada. Se ha sostenido que una concepción no reductivista de la causalidad, sin embargo, permitiría explicar adecuadamente cómo es que el agente sí es causa de sus decisiones libres y está en 'control' de ellas. No parece que sea decisivo para esta cuestión si el agente, por sí mismo, es causa de la decisión libre, o si la causa de la decisión libre es un evento que involucra al agente. Una concepción no reductivista de la causalidad puede ser motivada de un modo independiente, por lo demás.

Se considera, en segundo lugar, si es que el agente de una decisión libre ha tomado tal decisión por 'suerte'. Se ha sostenido que, aunque hay contextos en los que la baja probabilidad de un resultado hace que no pueda ser imputado a la acción de un agente que estaría en control de él, esto no es aplicable a una decisión libre. Realmente, por lo tanto, no hay dificultad para concebir la libertad de la voluntad con vínculos causales no deterministas. 


\section{REFERENCIAS}

-Chisholm, R. (1982). Human Freedom and the Self. En G. Watson (Ed.), Free Will (págs. 24-35). Oxford: Oxford University Press.

-Clarke, R. (2008). Incompatibilist (Nondeterministic) Theories of Free Will. (R. Zalta, Ed.) Recuperado el 23 de Enero de 2009, de Stanford Encyclopedia of Philosophy: http://plato.stanford.edu/entries/incompatibilism-theories.

-Collins, J., Hall, N. \& Paul, L. A. (2004). Counterfactuals and Causation: History, Problems, and Prospects. En J. Collins, N. Hall \& L. A. Paul (Eds.), Causation and Counterfactuals (págs. 1-57). Cambridge, Mass.: MIT Press.

-Denzinger, H. (1955). Enchiridion Symbolorum et Definitionum. (D. Ruiz, Trad.). Barcelona: Herder.

-Kim, J. (1993). Supervenience and Mind. Cambridge: Cambridge University Press.

-Lewis, D. (1986). Philosophical Papers. (Vol. II). Oxford: Oxford University Press.

-Lewis, D. (1999) (Ed.). Papers in Metaphysics and Epistemology. Cambridge: Cambridge University Press.

-Lewis, D. \& Langton, R. (1999). Defining 'Intrinsic'. En D. Lewis (Ed.), Papers in Metaphysics and Epistemology (págs. 116-132). Cambridge: Cambridge University Press.

-Tooley, M. (1987). Causation. A Realist Approach. Oxford: Clarendon Press.

-Van Inwagen, P. (1975). The Incompatibility of Free Will and Determinism. En G. Watson (Ed.), Free Will (págs. 46-58). Oxford: Oxford University Press.

-Van Inwagen, P. (1983). An Essay on Free Will. Oxford: Clarendon Press.

-Van Inwagen, P. (2008). The Mystery of Metaphysical Freedom. En P. van Inwagen \& D. W. Zimmerman (Eds.), Metaphysics. The Big Questions (págs. 456-465). Oxford: Blackwell.

-Watson, G. (1982) (Ed.). Free Will. Oxford: Oxford University Press.

Sumario: Introducción; 1. ¿Incompatibilidad de la libertad con el indeterminismo?; 2. Relaciones causales primitivas; 3. Suerte; Conclusiones; Referencias. 